\title{
Macro- and microstructural alterations of the subcortical structures in episodic
}

cluster headache

András Király ${ }^{1}$ MD, Nikoletta Szabó ${ }^{1,2}$ MD, PhD, Árpád Párdutz ${ }^{1}$ MD, PhD, Eszter Tóth ${ }^{1}$ MD, János Tajti ${ }^{1}$ MD, PhD, Gergő Csete ${ }^{1}$ MD, Péter Faragó ${ }^{1}$ MD, Péter Bodnár ${ }^{5}$, Délia Szok ${ }^{1}$ MD, PhD, Bernadett Tuka ${ }^{1,4} \mathrm{PhD}$, Éva Pálinkás MD ${ }^{6}$, Csaba Ertsey ${ }^{3} \mathrm{MD}, \mathrm{PhD}^{3}$, László Vécsei ${ }^{1,4} \mathrm{MD}, \mathrm{PhD}$, Zsigmond Tamás Kincses ${ }^{1,2} \mathrm{MD}$, PhD

${ }^{1}$ Department of Neurology, Albert Szent-Györgyi Clinical Center, University of Szeged, Szeged, Hungary

${ }^{2}$ International Clinical Research Center, St. Anne's University Hospital Brno, Brno, Czech Republic

${ }^{3}$ Department of Neurology, Semmelweis University, Budapest, Hungary

${ }^{4}$ MTA-SZTE Neuroscience Research Group, Szeged, Hungary

${ }^{5}$ Department of Image Processing and Computer Graphics, Faculty of Science and Informatics, Szeged, Hungary

${ }^{6}$ Bacs-Kiskun County Hospital, Kecskemét, Hungary

Key-words: clinical parameters, bootstrapping, DTI, interictal, laterality

Number of pages: 13

Number of tables: 5

Corresponding author:

Zsigmond Tamás Kincses MD, PhD

Neuroimaging Research Group

Department of Neurology

Albert Szent-Györgyi Clinical Center

University of Szeged

Semmelweis u. 6

H-6725 Szeged

HUNGARY

e-mail: kincses.zsigmond.tamas@med.u-szeged.hu

www: www.nepsy.szote.u-szeged.hu/ kincsesz 


\section{Abstract}

Background: Previous functional and structural imaging studies revealed that subcortical structures have key a role in pain processing. The recurring painful episodes might trigger maladaptive plasticity or alternatively degenerative processes that might be detected by MRI as changes in size or microstructure. In the current investigation we aimed to identify the macro- and microstructural alterations of the subcortical structures in episodic cluster headache.

Methods: High-resolution T1-weighted and diffusion-weighted MRI images with 60 gradient directions were acquired from 22 patients with cluster headache and 94 healthy controls. Surface-based segmentation analysis was used to measure volume of the subcortical nuclei and mean diffusion parameters (fractional anisotropy, mean, radial and axial diffusivity) were determined for these structures. In order to understand whether the size and diffusion parameters could be investigated in a headache lateralised manner, first the asymmetry of the size and diffusion parameters of the subcortical structures was analysed. Volumes and diffusion parameters were compared between groups and correlated with the cumulative number of headache days. To account for the different size of the patient and control group a bootstrap approach was used to investigate the stability of the findings.

Results: A significant lateralisation of the size (caudate, putamen and thalamus) and the diffusion parameters of the subcortical structures were found in normal controls.

In cluster headache patients the mean fractional anisotropy of the right amygdalae, the mean axial and mean diffusivity of the right caudate nucleus and the radial diffusivity of the right pallidum was higher. The mean anisotropy of the right pallidum was lower in patients.

Conclusion: The analysis of the pathology in the subcortical structures in episodic cluster headache reveals important features of the disease, which might allow a deeper insight into the pathomechanism of the pain processing in this headache condition. 


\section{Introduction}

Episodic cluster headache $(\mathrm{CH})$ is a primary headache disorder with the prominent feature of extremely severe unilateral, periorbital headache attacks accompanied by ipsilateral autonomic symptoms and occurring in clusters usually lasting some weeks, followed by much longer headache-free periods $(1,2)$.

The basal ganglia and generally the subcortical structures were recently proposed to have a central role in nociception. The facts that they receive input directly from the spinal cord and also via the thalamus and exhibit connections with various cortical regions involved in pain processing suggest that this system is ideal for the integration of various aspects of pain-related information (for an in-depth review, see (3) and (4)). Furthermore, it has been shown that basal ganglia structures are activated during painful stimuli (5) and putaminal and pallidal neurons are able to encode the intensity of noxious thermal stimuli (6). There are opioid receptors in the striatum, and nociceptive neurones have also been found (7). The nociceptive neurones of the spinal cord project directly to the globus pallidus, amygdala and hypothalamus (8). Moreover, the activation of the striatum correlate with the variability in pain sensation (9). Putaminal lesions were shown to alter the nociceptive information processing (10). Neuroimaging studies have demonstrated structural alterations in the striatum in chronic pain syndromes (11). In migraine, a larger caudate nucleus was observed in patients who displayed a high attack frequency (12). Imaging studies have also indicated the involvement of the subcortical structures in $\mathrm{CH}$. Atrophy of the thalamus and caudate nucleus has been reported in $\mathrm{CH}$ (13).

Disease related structural alterations could possibly be captured at the macrostructural level as size changes and the highly organized microstructure of the subcortical nuclei makes them readily available to study with diffusion weighted MRI. The two features might show parallel alterations, but since the histological background of the diffusion alterations in the grey matter is not well understood independency of the micro- and macrostructural alterations may also occur.

In the current study, we set out to investigate volumetric changes in $\mathrm{CH}$ by an approach highly tuned for the detection of subcortical volume changes (FIRST (14)). Furthermore, we made use of the highly organized microstructure of subcortical nuclei to investigate diffusion in the segmented nuclei. We also hypothesised that not all subcortical 
structures were affected by the disease at the same rate and we also wanted to analyse the co-occurrence of the micro- and macrostructural alterations in $\mathrm{CH}$.

We expected two possible outcomes: (i) Repetitive painful attacks might induce maladaptive plasticity in pain related cortical and subcortical structures. These changes might present in a volume augmentation and possibly in a more organized microstructure, as detected by diffusion tensor imaging. (ii) Alternatively, the recurrent severe pain and the concurrent pathological processes might induce degenerative changes, which present in the form of microstructural disintegration and atrophy.

Investigating the brain structures by pooling data as being contralateral or ipsilateral to the headache side is a frequently used approach in the headache literature to boost the number of observations. However, this approach can only be used if no lateralisation exists in healthy subjects. The lateralisation in healthy subjects was investigated by processing the MRI of a large group of volunteers, measured with the same parameters as those of the $\mathrm{CH}$ patients. The same large control group was used in all further comparisons, since any selection of a subgroup could cause a statistical bias. In order to account for the different size of the groups compared a bootstrap approach was used to confirm stability of the findings (15). The bootstrap approach takes random samples from each subgroup with the same size. By repeating the random sampling with replacement by a large number of times the bootstrap approach is not only giving a statistical estimator of the model, but the stability of that estimator can also be evaluated.

\section{Methods}

\subsection{Participants}

Twenty-seven patients were recruited into the study. Five patients were excluded due to comorbidity (depression, benign tumour), cessation of pain after tooth extraction (e.g. secondary cluster headache), and structural abnormality on the MRI scans. Finally, twenty-two patients were included (mean age: $38.10 \pm 11.33$, male: 19). Inclusion criteria for the $\mathrm{CH}$ patients were: $18-80$ years of age, primary $\mathrm{CH}$ according to The International Headache Society diagnostic criteria (1), no interval therapy for the $\mathrm{CH}$, no accompanying neurological (including other primary headache disorders and pain conditions) or psychiatric disease, no regular neuro-psychiatric medication, negative 
routine MRI scan. Special attention was paid to the exclusion of depression, for which the Hamilton questionnaire was used (>16 points was exclusion criterion) (16). The MRI acquisitions were carried out in the interictal period (at least 1 month after the last headache episode). There were 12 left-headache-sided (LHS-CH) and 10 right-headachesided patients $(\mathrm{RHS}-\mathrm{CH})$ in the $\mathrm{CH}$ group. All the participants were right handed. Clinical variables, such as disease duration, time between bouts and average length of bouts were recorded for all patients. Furthermore, cumulative number of headache days - that is the total number of days the patient had experienced cluster headache over his/her entire life - was estimated for all the patients.

94 healthy individuals (mean age: $32.59 \pm 10.43$, male: 50) were included in the study. Inclusion criteria for the controls were: 18-80 years of age, no history of neurological (including primary headaches, other pain conditions) or psychiatric diseases. All the participants were right handed.

Demographic data and the corresponding p-values are presented in Table 1a and $\mathbf{1 b .}$

The study was approved by the Ethics Committee of the University of Szeged (authority number: 87/2009), and all the subjects provided their written informed consent.

\subsection{Image acquisition}

MR image acquisition took place at least one month after the end of the last headache bout. Imaging was carried out with a 1.5 T GE Signa Excite MRI scanner. High-resolution T1-weighted images (3D IR-FSPGR: TR/TE/TI: 10.3/4.2/450 ms, flip angle: $15^{\circ}$, ASSET: 2, FOV: $25^{*} 25 \mathrm{~cm}$, matrix: $256^{*} 256$, slice thickness: $1 \mathrm{~mm}$ ) and 60 direction diffusionweighted images with 6 non-diffusion-weighted reference volumes (TE: 93.8 ms, TR: 13500 ms, matrix: 96x96, FOV: $23 \times 23 \mathrm{~cm}$, flip angle: $90^{\circ}$, in-plane resolution: $2.4 \times 2.4$ $\mathrm{mm}$, which was resampled to a $0.89 \times 0.89 \mathrm{~mm}$ by the scanner, slice thickness: $2.4 \mathrm{~mm}$, b: 1000s/m², NEX: 2, ASSET) were recorded.

\subsection{Image processing}

Data were processed by means of the tools from the FMRIB Software Library (FSL, version 5.0; Oxford Centre for Functional MRI of the Brain (FMRIB), UK; www.fmrib.ox.ac.uk/fsl). 


\subsubsection{Partial brain volumes of the brain and subcortical structures}

For comparisons of the volumes of the subcortical structures between the groups, FIRST analysis was used (14). FIRST is a model-based segmentation/registration tool. It uses deformable surface meshes specific to subcortical structures (amygdala, caudate nucleus, hippocampus, pallidum, putamen and thalamus). Based on learned models, FIRST searches through linear combinations of shape modes of variation for the most probable shape instance given the observed intensities in a T1-weighted image. The nucleus accumbens was not investigated because of the inappropriate segmentation.

Partial brain volumes (total brain volume, total grey matter, white matter, peripheral grey matter [cortex] and ventricular cerebrospinal fluid), with and without normalization for the intracranial volume, were compared between the two groups. The intracranial volume and the grey and white matter volumes were estimated by SIENAX $(17,18)$, part of FSL $(19)$. SIENAX starts by extracting brain and skull images from the single whole-head input data (20). The brain image is then affine-registered to MNI152 space $(21,22)$ (using the skull image to determine the registration scaling); this is primarily carried out in order to obtain the volumetric scaling factor (vscale), to be used as normalization for head size. Next, tissue-type segmentation with partial volume calculation is carried out (23) in order to calculate the total volume of brain tissue (including separate estimates of the volumes of the grey and the white matter), and the grey and white matter partial volumes.

\subsubsection{Diffusion parameters of the basal ganglia}

In order to evaluate the internal microstructure of the subcortical structures, diffusion parameters were estimated for each of the segmented subcortical structures and compared between the two groups. The diffusion data were corrected for eddy currents and movement artefacts by 12 degrees of freedom affine linear registration to the first non-diffusion-weighted reference image (22). Diffusion tensors at each voxel were fitted by the algorithm included in the Diffusion Toolbox (FDT) of the FMRIB's Software Library (FSL v. 4.0, www.fmrib.ox.ac.uk/fsl; (19)). Fractional anisotropy (FA), mean diffusivity (MD), and diffusivity parallel (AD, axial) and perpendicular (RD, radial) to the principal diffusion direction were computed for the whole brain. The binary masks of subcortical structures segmented by FIRST analysis of each subject were registered (FLIRT) to the subjects' own DWI images with 6 DOF. The transformed masks were 
thresholded at 0.5 and binarised again. All registered images then were checked visually and corrected so as not to contain parts of ventricles or white matter tracts close to the subcortical structures. Mean diffusion parameters were calculated under the masked areas.

Furthermore, the trigeminal nerves were identified in the axial plane using colour orientation maps created by overlaying the principal eigenvector image (V1) over the FA map. Then a 15 voxel sized ROIs were manually drawn on the root entry zone of the trigeminal nerves. Maximum and the mean of the diffusion parameters were estimated.

\subsection{Statistical analysis}

\subsubsection{Lateralisation in healthy subjects}

Left-right ratio of the size and diffusion parameters of the subcortical structures was estimated as the ratio of the left and right side parameters (1 meaning no lateralisation, numbers higher than 1 indicating higher parameters on the left and numbers lower than 1 indicating higher parameters on the right). One sample t-test was used to test lateralisation.

\subsubsection{Alteration of the size and diffusion parameters of the subcortical structures in $\mathrm{CH}$}

The group differences $\left(\mathrm{t}_{0}, \mathrm{p}_{0}\right)$ were evaluated by using standard general linear model, where the model was encoding group membership. While our analysis in healthy controls found no gender or age effect, these two variables were included in the analysis to account for the theoretical effect. The solution of the regression model was estimated by ordinary least squares approach. Since the number of subjects in the control and patient groups differed significantly a bootstrap approach was used to confirm the stability of the findings (15). 5000 bootstrap samples were randomly drawn from each group by the size of the patient population $(n=22)$ ensuring that the size of the two compared groups are always the same. The parameters $\left(t_{B}\right)$ of the GLM model were estimated for every bootstrap samples $\left(t_{B 1}-t_{B 5000}\right)$, then the mean of it was calculated $(\hat{t})$. The confidence interval for the bootstrapped t-values $(\hat{t}(C I))$ was used to perform an approximate two-sided test of a null hypothesis of the form:

$H_{0}: t_{0} \in \hat{t}(C I)$. 
The null hypothesis was to be rejected, and hence the original, non-bootstrapped parameter $\left(t_{0}\right)$ was not from the bootstrapped distribution on the significance level of 0.05 if $t_{0}$ lies outside the two-tailed $(1-\alpha)$ confidence interval $\hat{t}(C I)$.

A similar approach was used for the correlations between clinical variables, volumes and diffusion parameters (the correlation coefficient, $r$ was used in the bootstrap resampling).

\section{Results}

\subsection{Asymmetry of the size and diffusion parameters of the subcortical structures in healthy subjects}

The laterality of the size and the diffusion parameters of the subcortical structures were investigated in 94 healthy control subjects.

Head size normalized volumes of the right caudate nucleus, left putamen and left thalamus were significantly higher than the contralateral pair of these structures.

The FA of the left amygdala, caudate nucleus, putamen and right pallidum was higher than the contralateral pair of these structures. AD and MD of all right side structures except thalamus - were higher than in the contralateral structures. RD of the right amygdala, caudate nucleus, pallidum, putamen and the left hippocampus were higher than in the contralateral structures. (Table 2.)

These results indicate that there is a significant lateralisation of the size and diffusion parameters in healthy subjects. Consequently, pooled parameters according to the headache side cannot be used in the group comparisons.

\subsection{Alteration of the size and diffusion parameters of the subcortical structures in $\mathrm{CH}$}

The GLM analysis showed that the FA of the right amygdala was significantly higher in CH and $L H S-C H$ patients than in healthy subjects. A similar tendency was found in case of the left amygdala in RHS-CH patients. MD and RD of the right amygdala were higher in healthy subjects compared to the $\mathrm{CH}$ and $L H S$ - $C H$ patients. A similar tendency was found in $R H S-C H$ patients.

$\mathrm{AD}$ of the right caudate nucleus was higher in $\mathrm{CH}, \mathrm{RHS}-\mathrm{CH}$ and $L H S-C H$ patients as compared with healthy subjects. A similar tendency was found with MD and RD in $C H$ and $L H S-C H$ patients. 
In case of the right pallidum FA was lower in $\mathrm{CH}$ and $\mathrm{LHS}$ - $\mathrm{CH}$ patients. RD of the right pallidum showed tendency to be higher in $\mathrm{CH}$ and $\mathrm{LHS}-\mathrm{CH}$ patients than in healthy subjects. The head size normalized volume of the right pallidum was lower in $R H S-C H$ patients than in healthy subjects.

According to the diffusion parameters of the trigeminal root entry zone there were no significant differences between patients or controls, or between the ipsilateral and contralateral measures. Total brain volume or the grey and white matter volumes were not different between groups either.

Significance levels and bootstrap confidence intervals are presented in: Table 3.

\subsection{Correlation of the volumes and diffusion parameters with clinical parameters}

The head size normalised volume of the total brain and cortical grey matter showed positive correlation with the cumulative number of headache days in the $\mathrm{CH}$ patients. Similar correlation was found in the $L H S$-CH patients. Also total gray matter and white matter showed similar, but not significant tendency in the $\mathrm{CH}$ patients.

The head size normalized volume of the left and right hippocampus and right caudatus showed positive, the $\mathrm{AD}$ of the left and right thalamus, the MD and $\mathrm{RD}$ of the left hippocampus showed negative correlation with the cumulative number of headache days in the $\mathrm{CH}$ patients.

Head size normalized volume of the left pallidum, left and right thalamus showed positive, the $\mathrm{AD}$ of the left hippocampus, the MD of the left pallidum showed negative correlation with the cumulative number of headache days in the RHS-CH patients.

The head size normalized volume of the left hippocampus showed positive correlation with the cumulative number of headache days in the $L H S$ - $C H$ patients.

Significance levels and bootstrap confidence intervals are presented in: Table 4.

\section{Discussion}

In this MRI study we demonstrated an altered interictal microstructure of the subcortical structures in $\mathrm{CH}$. Some of these microstructural changes were dependent on the lifetime disease burden. Demonstrating the lateralisation of diffusion parameters of the subcortical structures in a large cohort of healthy subjects, we showed that the 
headache side dependent pooling of the data to boost the number of observations is not recommended.

In neuroimaging studies investigating unilateral processes, such as in $\mathrm{CH}$ and migraine to boost the number of observations, it is common to flip the data about the midsaggital axis to have hemispheres/structures aligned according to the headache side (24-26). This approach is used despite it was shown that there is a normal asymmetry in the white matter diffusion parameters (27). Most consistent asymmetry was found in the arcuate fasciculus and in the cingulum (27-29). Similarly, diffusion parameters of the subcortical structures were reported to be asymmetric in healthy subjects (30). While Fabiano's findings are important and consistent with our findings, in their study the mean of diffusivity from only three directions were examined in a small cohort of healthy subjects and circular regions of interests were used in some of the structures (30). In our study we used a larger cohort of healthy subjects and found asymmetries of various diffusion parameters estimated from diffusion measurements from 60 different directions. Based on these results we concluded that we cannot pool data from the different hemispheres of $\mathrm{CH}$ patients. In our analysis we treated $\mathrm{CH}$ patients as one group regardless of the side of the headache. Only as a secondary test we compared the RSH-CH and LSH-CH patients to healthy subjects. Because of the small size of the groups, the results of these later comparisons have to be handled cautiously even though the stability of the findings were evaluated by a bootstrap sampling. Furthermore, it has to be pointed out that the size of the subgroups of $\mathrm{CH}$ patients in our analysis does not allow us to investigate headache side dependent alterations of parameters or alterations of lateralisation of these parameters.

Macro- and microstructural alterations are well-known features of pain-related disorders $(24,31)$. As regarding $\mathrm{CH}$, the seminal paper by May et al. (32) described an increased grey matter volume in the inferior, posterior hypothalamus in $\mathrm{CH}$, but no other changes in grey matter density in either the cortex or the subcortical structures. In contrast, another VBM analysis indicated a decreased grey matter volume in the right thalamus, in the head of the right caudate nucleus and in several pain-processing-related cortical structures, such as the right precentral gyrus, right posterior cingulate cortex, bilateral middle frontal gyrus, right middle temporal gyrus, left inferior parietal lobule and left insula (13). Importantly, the decrease in the grey matter volume did not depend on the side of the pain. While slightly smaller subcortical structures were found, no 
subcortical volume alteration was identified with our surface-based analysis approach (except the right pallidum that was smaller in $R H S-C H$ than in healthy). A recent VBM style investigation also called attention to that the cortical and subcortical volumetric alterations are dynamic in relation to the pain state (e.g. in an out of bouts and in chronic $\mathrm{CH}$ ) (25).

Alterations in the diffusion parameters in the white matter in primary headache disorders such as migraine and $\mathrm{CH}$ are already known $(24,31)$, but the microstructural alterations in the subcortical structures described here have not been investigated previously in $\mathrm{CH}$. Only a few studies dealt with the pain-related changes in the diffusion parameters in the subcortical structures. An increased fractional anisotropy has been reported in the thalamus contralateral to the affected body side in multiple sclerosis patients with extremity pain (33). In irritable bowel syndrome decreased FA was found in thalamic regions, a reduced MD in the globus pallidus and a higher MD were described in the thalamus (34). The apparent diffusion coefficient (ADC) in the red nucleus of patients with migraine was shown to be increased relative to healthy subjects (35). In migraineurs without aura, FA was reported to be higher and MD to be lower in the bilateral thalami as compared to controls in the interictal phase (36). The diffusivity parameters have also been found to be similar to those in controls in the ictal phase (36). While the diffusion parameters of the trigeminal nerve or the root entry zone have been investigated in trigeminal neuralgia (37), there have been no prior investigations published in $\mathrm{CH}$. Our results did not find $\mathrm{CH}$ specific alterations of the diffusion parameters of the trigeminal root entry zone, but one possible source of this negative result might be the high variability in the data. In average the neural and vascular structures of the prepontine fossa range between 0.5 and $3.0 \mathrm{~mm}$ in diameter. This small diameter has to be considered in diffusion-tensor imaging studies in terms of the limited spatial resolution of the sequences used (38).

The FA of the bilateral amygdala was found increased in our study. Amygdala is known for being an important centre of emotional and affective aspects of pain. It is an important hub in processing of the noxious stimuli and it was shown to have structural and functional connections to cortical and subcortical structures involved in pain processing $(39,40)$. It was shown by several studies that there is a significant pain related plasticity in the amygdala in chronic pain conditions and amygdalar plasticity was suggested to be the key factor in the establishment of fear memory $(41,42)$. We 
propose that the increased FA in $\mathrm{CH}$ patients might have a similar background. Interestingly, the pain related activation of amygdala is heavily lateralised to the right (43), but no such lateralisation of diffusion parameters or size was found in our patients. While it is known that basal ganglia structures - such as the pallidum and caudate nucleus, which was found to have altered diffusion parameters in $\mathrm{CH}$ in our study - are activated in response to painful stimuli (5) and structural alterations are related to chronic pain conditions (11) the exact role of these structures in the processing of noxious stimulus is not well understood yet.

In a prior study we have shown diffusion parameter alterations in the white matter of $\mathrm{CH}$ patients (31). In the white matter, the pattern of diffusion parameter alterations may be related to the underlying pathological microstructural changes. In mouse models (44), the changes observed in $\mathrm{AD}$ and $\mathrm{RD}$ have been suggested to relate to axon or myelin damage, respectively. However, the background of the diffusion parameter changes in the subcortical grey matter is not so clear. Nevertheless, the subcortical grey nuclei have a well-defined microstructure, which - as we have shown - is altered by $\mathrm{CH}$. There can be a few processes leading to the alteration of the diffusion parameters of the subcortical structures. The contribution of the extracellular and intracellular water fraction to the diffusion signal is not even. A combination of diffusion parameter changes could be explained by the change of the ratio of the extra and intracellular water fractions (e.g.: global shrinkage of the structure or intracellular oedema) or for example protein deposits limiting the water diffusion. Alterations can also be caused by the change of myelin or axon content or fibre orientation.

Recent studies suggest that neurodegeneration could result in diffusion changes in related basal ganglia. In movement disorders, in which the basal ganglia have a crucial role in pathomechanism, altered diffusivity parameters were detected (in Huntington's disease: (45); in Parkinson's disease: (46); in blepharospasm: (47)). We consider that this pattern points to the co-occurrence of degenerative changes, presenting in the form of microstructural disintegration and compensatory or pain-related maladaptive plastic changes in $\mathrm{CH}$.

\section{Limitations}

This analysis has some essential drawbacks. First of all, it is a cross-sectional assessment of the focal shrinkage of the subcortical and cortical volumes, also we do not have exact 
information about the time elapsed from the last attack. A further drawback is the relatively low power of the secondary analyses (RHS-CH and LHS-CH) due to the limited number of patients. Finally, while only patients with no history of other neurological conditions were recruited/involved, we did not have any information about additional risk factors (e.g. diabetes mellitus) that the patients may have had. In order to acquire an in-depth understanding of the dynamics of brain atrophy and diffusion parameters longitudinal studies are needed. Given the central role of the hypothalamus in $\mathrm{CH}$ it would be crucial to investigate its size and microstructure. Unfortunately, our approach in its current form is not able to segment the hypothalamus, segmentation of the structure being limited by the low difference in intensity from the surrounding structures.

\section{Acknowledgements}

The authors declare that there are no conflicts of interest. The study was supported by the „Neuroscience Research Group of the Hungarian Academy of Sciences and University of Szeged”, the core facility MAFIL of CEITEC supported by the MEYS CR (LM2015062 Czech-BioImaging), the project FNUSA-ICRC (no. CZ.1.05/1.1.00/02.0123) from the European Regional Development Fund, by European Union - project ICRC-ERA-HumanBridge (No. 316345), the National Brain Research Program (Grant No. KTIA_13_NAP-A-II/20.) and an OTKA [PD 104715] grant. Dr. Szabó was supported by the Bolyai Scholarship Program of the Hungarian Academy of Sciences. 


\section{References}

[1] Headache Classification Subcommittee of the International Headache S. The International Classification of Headache Disorders: 2nd edition. Cephalalgia : an international journal of headache. 2004;24 Suppl 1:9-160.

[2] Russell MB. Epidemiology and genetics of cluster headache. Lancet neurology. 2004;3:279-83.

[3] Borsook D, Upadhyay J, Chudler EH, Becerra L. A key role of the basal ganglia in pain and analgesia--insights gained through human functional imaging. Molecular pain. 2010;6:27.

[4] Juri C, Rodriguez-Oroz M, Obeso JA. The pathophysiological basis of sensory disturbances in Parkinson's disease. Journal of the neurological sciences. 2010;289:60-5. [5] Bingel U, Glascher J, Weiller C, Buchel C. Somatotopic representation of nociceptive information in the putamen: an event-related fMRI study. Cereb Cortex. 2004;14:1340-5.

[6] Chudler EH. Response properties of neurons in the caudate-putamen and globus pallidus to noxious and non-noxious thermal stimulation in anesthetized rats. Brain Res. 1998;812:283-8.

[7] Baumgartner U, Buchholz HG, Bellosevich A, Magerl W, Siessmeier T, Rolke R, et al. High opiate receptor binding potential in the human lateral pain system. Neuroimage. 2006;30:692-9.

[8] Braz JM, Nassar MA, Wood JN, Basbaum AI. Parallel "pain" pathways arise from subpopulations of primary afferent nociceptor. Neuron. 2005;47:787-93.

[9] Hagelberg N, Jaaskelainen SK, Martikainen IK, Mansikka H, Forssell H, Scheinin H, et al. Striatal dopamine D2 receptors in modulation of pain in humans: a review. Eur J Pharmacol. 2004;500:187-92.

[10] Starr CJ, Sawaki L, Wittenberg GF, Burdette JH, Oshiro Y, Quevedo AS, et al. The contribution of the putamen to sensory aspects of pain: insights from structural connectivity and brain lesions. Brain. 2011;134:1987-2004.

[11] Schmidt-Wilcke T, Leinisch E, Ganssbauer S, Draganski B, Bogdahn U, Altmeppen J, May A. Affective components and intensity of pain correlate with structural differences in gray matter in chronic back pain patients. Pain. 2006;125:89-97.

[12] Maleki N, Becerra L, Nutile L, Pendse G, Brawn J, Bigal M, et al. Migraine attacks the Basal Ganglia. Molecular pain. 2011;7:71.

[13] Absinta M, Rocca MA, Colombo B, Falini A, Comi G, Filippi M. Selective decreased grey matter volume of the pain-matrix network in cluster headache. Cephalalgia : an international journal of headache. 2012;32:109-15.

[14] Patenaude B, Smith SM, Kennedy DN, Jenkinson M. A Bayesian model of shape and appearance for subcortical brain segmentation. Neuroimage. 2011;56:907-22.

[15] Efron B. Missing Data, Imputation, and the Bootstrap. J Am Stat Assoc. 1994;89:463-75.

[16] Hamilton M. A rating scale for depression. Journal of neurology, neurosurgery, and psychiatry. 1960;23:56-62.

[17] Smith SM, De Stefano N, Jenkinson M, Matthews PM. Normalized accurate measurement of longitudinal brain change. Journal of computer assisted tomography. 2001;25:466-75. 
[18] Smith SM, Zhang Y, Jenkinson M, Chen J, Matthews PM, Federico A, De Stefano N. Accurate, robust, and automated longitudinal and cross-sectional brain change analysis. Neuroimage. 2002;17:479-89.

[19] Smith SM, Jenkinson M, Woolrich MW, Beckmann CF, Behrens TE, Johansen-Berg $\mathrm{H}$, et al. Advances in functional and structural MR image analysis and implementation as FSL. Neuroimage. 2004;23 Suppl 1:S208-19.

[20] Smith SM. Fast robust automated brain extraction. Human brain mapping. 2002;17:143-55.

[21] Jenkinson M, Bannister P, Brady M, Smith S. Improved optimization for the robust and accurate linear registration and motion correction of brain images. Neuroimage. 2002;17:825-41.

[22] Jenkinson M, Smith S. A global optimisation method for robust affine registration of brain images. Medical image analysis. 2001;5:143-56.

[23] Zhang Y, Brady M, Smith S. Segmentation of brain MR images through a hidden Markov random field model and the expectation-maximization algorithm. IEEE transactions on medical imaging. 2001;20:45-57.

[24] Szabo N, Kincses ZT, Pardutz A, Tajti J, Szok D, Tuka B, et al. White matter microstructural alterations in migraine: a diffusion-weighted MRI study. Pain. 2012;153:651-6.

[25] Naegel S, Holle D, Desmarattes N, Theysohn N, Diener HC, Katsarava Z, Obermann M. Cortical plasticity in episodic and chronic cluster headache. NeuroImage Clinical. 2014;6:415-23.

[26] Teepker M, Menzler K, Belke M, Heverhagen JT, Voelker M, Mylius V, et al. Diffusion tensor imaging in episodic cluster headache. Headache. 2012;52:274-82.

[27] Takao H, Hayashi N, Ohtomo K. White matter asymmetry in healthy individuals: a diffusion tensor imaging study using tract-based spatial statistics. Neuroscience. 2011;193:291-9.

[28] Buchel C, Raedler T, Sommer M, Sach M, Weiller C, Koch MA. White matter asymmetry in the human brain: a diffusion tensor MRI study. Cereb Cortex. 2004;14:945-51.

[29] Gong G, Jiang T, Zhu C, Zang Y, He Y, Xie S, Xiao J. Side and handedness effects on the cingulum from diffusion tensor imaging. Neuroreport. 2005;16:1701-5.

[30] Fabiano AJ, Horsfield MA, Bakshi R. Interhemispheric asymmetry of brain diffusivity in normal individuals: a diffusion-weighted MR imaging study. AJNR Am J Neuroradiol. 2005;26:1089-94.

[31] Szabo N, Kincses ZT, Pardutz A, Toth E, Szok D, Csete G, Vecsei L. White matter disintegration in cluster headache. The journal of headache and pain. 2013;14:64.

[32] May A, Goadsby PJ. Hypothalamic involvement and activation in cluster headache. Current pain and headache reports. 2001;5:60-6.

[33] Deppe M, Muller D, Kugel H, Ruck T, Wiendl H, Meuth SG. DTI detects water diffusion abnormalities in the thalamus that correlate with an extremity pain episode in a patient with multiple sclerosis. NeuroImage : clinical. 2013;2:258-62.

[34] Ellingson BM, Mayer E, Harris RJ, Ashe-McNally C, Naliboff BD, Labus JS, Tillisch $\mathrm{K}$. Diffusion tensor imaging detects microstructural reorganization in the brain associated with chronic irritable bowel syndrome. Pain. 2013;154:1528-41.

[35] Kara B, Kiyat Atamer A, Onat L, Ulusoy L, Mutlu A, Sirvanci M. DTI Findings During Spontaneous Migraine Attacks. Clinical neuroradiology. 2013;23:31-6.

[36] Coppola G, Tinelli E, Lepre C, Iacovelli E, Di Lorenzo C, Di Lorenzo G, et al. Dynamic changes in thalamic microstructure of migraine without aura patients: a 
diffusion tensor magnetic resonance imaging study. European journal of neurology : the official journal of the European Federation of Neurological Societies. 2014;21:287-e13. [37] Lutz J, Thon N, Stahl R, Lummel N, Tonn JC, Linn J, Mehrkens JH. Microstructural alterations in trigeminal neuralgia determined by diffusion tensor imaging are independent of symptom duration, severity, and type of neurovascular conflict. J Neurosurg. 2015:1-8.

[38] Yousry I, Camelio S, Schmid UD, Horsfield MA, Wiesmann M, Brückmann H, Yousry TA. Visualization of cranial nerves I-XII: value of 3D CISS and T2-weighted FSE sequences. Eur Radiol. 2000;10:1061-7.

[39] Bach DR, Behrens TE, Garrido L, Weiskopf N, Dolan RJ. Deep and superficial amygdala nuclei projections revealed in vivo by probabilistic tractography. J Neurosci. 2011;31:618-23.

[40] Mishra A, Rogers BP, Chen LM, Gore JC. Functional connectivity-based parcellation of amygdala using self-organized mapping: a data driven approach. Human brain mapping. 2014;35:1247-60.

[41] Ji G, Neugebauer V. Differential effects of CRF1 and CRF2 receptor antagonists on pain-related sensitization of neurons in the central nucleus of the amygdala. J Neurophysiol. 2007;97:3893-904.

[42] Tsvetkov E, Carlezon WA, Benes FM, Kandel ER, Bolshakov VY. Fear conditioning occludes LTP-induced presynaptic enhancement of synaptic transmission in the cortical pathway to the lateral amygdala. Neuron. 2002;34:289-300.

[43] Ji G, Neugebauer V. Hemispheric lateralization of pain processing by amygdala neurons. J Neurophysiol. 2009;102:2253-64.

[44] Song SK, Yoshino J, Le TQ, Lin SJ, Sun SW, Cross AH, Armstrong RC. Demyelination increases radial diffusivity in corpus callosum of mouse brain. Neuroimage. 2005;26:132-40.

[45] Syka M, Keller J, Klempir J, Rulseh AM, Roth J, Jech R, et al. Correlation between Relaxometry and Diffusion Tensor Imaging in the Globus Pallidus of Huntington's Disease Patients. PloS one. 2015;10:e0118907.

[46] Kim HJ, Kim SJ, Kim HS, Choi CG, Kim N, Han S, et al. Alterations of mean diffusivity in brain white matter and deep gray matter in Parkinson's disease. Neuroscience letters. 2013;550:64-8.

[47] Blood AJ, Tuch DS, Makris N, Makhlouf ML, Sudarsky LR, Sharma N. White matter abnormalities in dystonia normalize after botulinum toxin treatment. Neuroreport. 2006;17:1251-5. 